\title{
Conformal-twisted product semi-slant submanifolds in globally conformal Kaehler manifolds
}

\author{
Sibel Gerdan Aydın*(i), Hakan Mete Taştan (D) \\ Department of Mathematics, Faculty of Science, İstanbul University, İstanbul 34134, Turkey
}

\begin{abstract}
We introduce the notion of conformal-twisted product submanifolds of the form ${ }_{f} M^{T} \times_{b} M^{\theta}$ and ${ }_{f} M^{\theta} \times_{b} M^{T}$, where $M^{T}$ is a holomorphic submanifold and $M^{\theta}$ is a proper slant submanifold of $M$ in a globally conformal Kaehler manifold and $f$ and $b$ are conformal factor and twisting function, respectively. We give necessary and sufficient conditions for proper semi-slant submanifold to be a locally conformal-twisted product for such submanifolds of the form ${ }_{f} M^{T} \times_{b} M^{\theta}$ and ${ }_{f} M^{\theta} \times_{b} M^{T}$. We establish a general inequality for the squared norm of second fundamental form of these types of submanifolds.
\end{abstract}

Mathematics Subject Classification (2020). 53C15, 53B20

Keywords. twisted product submanifold, holomorphic distribution, slant distribution, semi-slant submanifold, locally and globally conformal Kaehler manifold

\section{Introduction}

In differential geometry, one of the most intensively research areas is the theory of submanifolds. Actually, there are well-known classes of submanifolds such as holomorphic(invariant), totally real(anti-invariant) [29], CR- [1], semi-invariant [2], slant [6], semislant [17], etc. All classes are determined by the behavior of the almost complex or almost product structure of the ambient manifold.

Bishop and O Neill [3] introduced the notion of warped product of Riemannian manifolds to construct a large class of complete manifolds of negative curvature. This concept is also a generalization of the usual product of Riemannian manifolds. The theory of warped product submanifolds has been becoming a popular research area since Chen [7] studied the warped product CR-submanifolds in Kaehler manifolds. Most of the studies related to the theory of warped product submanifolds can be found in Chen's book[8].

Unlike the warped product submanifolds, the doubly warped product submanifolds have not been a so active research area. Perhaps this is because some well-known structures such as Kaehler, nearly Kaehler, locally product Riemannian and trans-Sasakian

\footnotetext{
*Corresponding Author.

Email addresses: sibel.gerdan@istanbul.edu.tr (S. Gerdan Aydın), hakmete@istanbul.edu.tr (H. M. Taştan)

Received: 29.09.2020; Accepted: 17.02.2021
} 
do not allow non-trivial doubly warped product submanifolds whose factors are holomorphic(invariant), totally real(anti-invariant) submanifolds (see, [16, 20, 22, 24]). However, doubly warped product CR-submanifolds were studied in locally conformal Kaehler manifolds in [15].

The non-existence of non-trivial warped product semi-slant submanifolds in Kaehlerian manifolds was proved by Şahin [19]. Indeed, there do not exist warped product semi-slant submanifolds in Kaehlerian manifolds in the form $M^{\theta} \times_{f} M^{T}$ and $M^{T} \times_{f} M^{\theta}$, where $M^{T}$ is a holomorphic and $M^{\theta}$ is a proper slant submanifold of the Kaehlerian manifold[19]. Moreover, Şahin [21] defined and studied warped product semi-slant submanifolds in the form $M^{\theta} \times_{f} M^{T}$ in locally product Riemannian manifolds contrary to Kaehlerian case, where $M^{T}$ is an invariant submanifold of the locally product Riemannian manifold. It was proved the non-existence of non-trivial warped product semi-slant submanifolds of the form $M^{T} \times_{f} M^{\theta}$ in a locally product Riemannian manifold in the same paper [21].

In [22], we defined two classes of doubly twisted products under the names of nearly doubly twisted products of type 1 and type 2 . In this article, we rename the nearly doubly twisted product of type 2 as conformal-twisted products.

Recently, Taştan and Tripathi [23] studied semi-slant submanifolds of locally conformal Kaehler manifolds. On the other hand, Matsumoto studied warped product semi-slant submanifolds in the forms $M^{\theta} \times_{f} M^{T}$ and $M^{T} \times_{f} M^{\theta}$ in locally conformal Kaehler manifolds in $[13,14]$. Inspired by these papers, we consider and study conformal-twisted product semi-slant submanifolds in globally conformal Kaehler manifolds and we give non-trivial examples for a conformal-twisted product proper semi-slant submanifold in globally conformal Kaehler manifold. We obtain some necessary and sufficient conditions for proper semi-slant submanifold to be locally conformal-twisted product for such submanifolds of the form ${ }_{f} M^{T} \times_{b} M^{\theta}$ and ${ }_{f} M^{\theta} \times_{b} M^{T}$. Moreover, we establish a general inequality for the squared norm of the second fundamental form of these types of submanifolds.

\section{Preliminiaries}

In this section, we recall the fundamental definitions and notions needed further study. Actually, in subsection 2.1, we give the definitions of doubly twisted and conformal-twisted products and in subsection 2.2, we will recall the definitions of locally and globally conformal Kaehler manifolds. The basic background for submanifolds of Riemannian manifolds will be presented in subsection 2.3.

\subsection{Conformal-twisted products}

Let $M_{1}$ and $M_{2}$ be Riemannian manifolds endowed with metric tensors $g_{1}$ and $g_{2}$, respectively and let $f$ and $b$ are positive smooth functions defined on $M_{1} \times M_{2}$. Then the doubly twisted product manifold [18] ${ }_{f} M_{1} \times{ }_{b} M_{2}$ is the product manifold $\bar{M}=M_{1} \times M_{2}$ equipped with metric $g$ given by

$$
g=f^{2} g_{1}+b^{2} g_{2} .
$$

Each function $f$ and $b$ is called a twisting function of the doubly twisted product $\left({ }_{f} M_{1} \times{ }_{b}\right.$ $\left.M_{2}, g\right)$.

If the twisting functions $f$ and $b$ only depend only on the points of $M_{1}$ and $M_{2}$ respectively, then $\left({ }_{f} M_{1} \times{ }_{b} M_{2}, g\right)$ is called doubly warped product manifold [25]. In which case, $f$ and $b$ are called warping functions of doubly warped product. 
Let $\left({ }_{f} M_{1} \times{ }_{b} M_{2}, g\right)$ be a doubly twisted product manifold. If $f=1$, then we get twisted product [5] $M_{1} \times_{b} M_{2}$ with twisting function $b$.

In addition, if the twisting function $b$ only depends only on the point of $M_{1}$, then $M_{1} \times_{b} M_{2}$ is called warped product [3] of $\left(M_{1}, g_{1}\right)$ and $\left(M_{2}, g_{2}\right)$ and the function $b$ is called warping function. If $b$ is a constant, then we get direct product manifold [8].

Let $\left(M_{1}, g_{1}\right)$ and $\left(M_{2}, g_{2}\right)$ be Riemannian manifolds and also let $f: M_{1} \rightarrow(0, \infty)$ and $b: M_{1} \times M_{2} \rightarrow(0, \infty)$ be smooth functions. The conformal-twisted product [22] is the product manifold $M_{1} \times M_{2}$ equipped with the metric tensor $g$ defined by

$$
g=\left(f \circ \pi_{1}\right)^{2} \pi_{1}^{*}\left(g_{1}\right)+\left(b \circ \pi_{2}\right)^{2} \pi_{2}^{*}\left(g_{2}\right),
$$

where $\pi_{1}$ and $\pi_{2}$ are canonical projections of $M_{1} \times M_{2}$ onto $M_{1}$ and $M_{2}$, respectively. For brevity in notation, we denote this Riemannian manifold $(M, g)$ by ${ }_{f} M_{1} \times_{b} M_{2}$. For a conformal-twisted product manifold, $f$ is called a conformal factor. In either case, the function $b$ is called a twisting function.

Let $\left({ }_{f} M_{1} \times{ }_{b} M_{2}, g\right)$ be a conformal-twisted product manifold with the Levi-Civita connection $\bar{\nabla}$ and $\nabla^{i}$ denote the Levi-Civita connection of ${ }_{f} M_{1} \times_{b} M_{2}$ and $M_{i}$ for $i \in\{1,2\}$, respectively. By usual convenience, we denote the set of lifts of vector fields on $M_{i}$ by $\mathcal{L}\left(M_{i}\right)$ and use the same notation for a vector field and for its lift. On the other hand, $\pi_{1}$ is an isometry and $\pi_{2}$ is a (positive) homothety, so they preserve the Levi-Civita connection. Thus, there is no confusion using the same notation for a connection on $M_{i}$ and for its pullback via $\pi_{i}$. Then, we have

$$
\begin{gathered}
\bar{\nabla}_{X} Y=\nabla_{X}^{1} Y+X(\ln f) Y+Y(\ln f) X-g(X, Y) \bar{\nabla} \ln f, \\
\bar{\nabla}_{V} X=\bar{\nabla}_{X} V=X(\ln b) V, \\
\bar{\nabla}_{U} V=\nabla_{U}^{2} V+U(\ln b) V+V(\ln b) U-g(U, V) \bar{\nabla} \ln b .
\end{gathered}
$$

for any $X, Y \in \mathcal{L}\left(M_{1}\right)$ and $U, V \in \mathcal{L}\left(M_{2}\right)$. The manifold $\left(M_{2}, g_{2}\right)$ is called a fiber of the conformal-twisted product and the manifold $\left(M_{1}, g_{1}\right)$ is called a base manifold of $\left({ }_{f} M_{1} \times_{b} M_{2}, g\right)$. It is seen that, the base manifold is totally geodesic from (2.2) while the fiber is totally umbilic from (2.4).

Remark 2.1. As seen from the definitions of doubly twisted product and conformaltwisted product, the conformal-twisted product case is a special case of doubly twisted product. Indeed, in the case of conformal-twisted product, the covariant derivatives formulas $(2.2) \sim(2.4)$ are simpler than the case of doubly twisted product, see Lemma 2.1 of $[11]$.

\subsection{Locally and globally conformal Kaehler manifolds}

Let $(\bar{M}, J, g)$ be a Hermitian manifold of dimension $2 m$. Then it is called a locally conformal Kaehler manifold (briefly l.c.K. manifold) [10], if each point of $p \in \bar{M}$ has an open neighborhood $\mathcal{U}$ with smooth function $\sigma: \mathcal{U} \rightarrow R$ such that $\tilde{g}=\left.e^{-\sigma} g\right|_{\mathfrak{u}}$ is a Kaehler metric on $\mathcal{U}$. If one choose $\mathcal{U}=\bar{M}$, then $(\bar{M}, J, g)$ is called a globally conformal Kaehler manifold (briefly g.c.K. manifold).

Theorem $2.2([10])$. Let $(\bar{M}, J, g)$ be a Hermitian manifold and let $\Omega$ be a 2 - form defined by $\Omega(\bar{X}, \bar{Y})=g(\bar{X}, J \bar{Y})$ for all vector fields $\bar{X}$ and $\bar{Y}$ in $\bar{M}$. Then $(\bar{M}, J, g)$ is a l.c.K. manifold if and only if there exists a globally defined $1-$ form $\omega$ such that

$$
d \Omega=\omega \wedge \Omega \quad \text { and } \quad d \omega=0 .
$$


The closed 1 - form $\omega$ is called the Lee form of the l.c.K. manifold $(\bar{M}, J, g)$. In addition, the manifold $(\bar{M}, J, g)$ is g.c.K., if its Lee form $\omega$ is also exact. In this case, we have $\omega=d \sigma$ [26]. The Lee vector field $B$ is defined by

$$
\omega(\bar{X})=g(B, \bar{X}),
$$

for any vector fields $\bar{X}$ on $\bar{M}$. One can see that, the globally conformal Kaehler case is a special case of the locally conformal Kaehler case. We denote by $\tilde{\nabla}$ (resp. $\bar{\nabla}$ ) the Levi-Civita connection on $\bar{M}$ with respect to $\tilde{g}=e^{-\sigma} g$ (resp. $g$ ). Then we have [10]

$$
\tilde{\nabla}_{\bar{X}} \bar{Y}=\bar{\nabla}_{\bar{X}} \bar{Y}-\frac{1}{2}\{\omega(\bar{X}) \bar{Y}+\omega(\bar{Y}) \bar{X}-g(\bar{X}, \bar{Y}) B\},
$$

for any vector fields $\bar{X}$ and $\bar{Y}$ on $\bar{M}$. The connection $\tilde{\nabla}$ is a torsionless linear connection on $\bar{M}$ which is called the Weyl connection of $g$. It is easy to see that the Weyl connection $\tilde{\nabla}$ satisfies the condition

$$
\tilde{\nabla} J=0 \text {. }
$$

For examples and more details on l.c.K. and g.c.K. manifolds we refer to [10].

Remark 2.3. Throughout this paper, we denote by $(\bar{M}, J, \omega, g)$ the g.c.K. manifold with the Lee form $\omega$.

\subsection{Submanifolds of Riemannian manifolds}

Let $M$ be an isometrically immersed submanifold in a Riemannian manifold $(\bar{M}, g)$. Let $\bar{\nabla}$ is the Levi-Civita connection of $\bar{M}$ with respect to the metric $g$ and let $\nabla$ and $\nabla^{\perp}$ be the induced, and induced normal connection on $M$, respectively. Then, for all $X, Y \in \Gamma(T M)$ and $Z \in \Gamma\left(T^{\perp} M\right)$, the Gauss and Weingarten formulas are given respectively by

$$
\begin{aligned}
& \bar{\nabla}_{X} Y=\nabla_{X} Y+h(X, Y), \\
& \bar{\nabla}_{X} Z=-A_{Z} X+\nabla_{X}^{\perp} Z,
\end{aligned}
$$

where $T M$ is the tangent bundle and $T^{\perp} M$ is the normal bundle of $M$ in $\bar{M}$. Additionally, $h$ is the second fundamental form of $M$ and $A_{Z}$ is the Weingarten endomorphism associated with $Z$. The second fundamental form $h$ and the shape operator $A$ are related by

$$
g(h(X, Y), Z)=g\left(A_{Z} X, Y\right) .
$$

The mean curvature vector field $H$ of $M$ is given by $H=\frac{1}{m}$ (trace $\left.h\right)$, where $\operatorname{dim}(M)=m$. We say that the submanifold $M$ is totally geodesic in $\bar{M}$ if $h=0$, and minimal if $H=0$. The submanifold $M$ is called totally umbilical if $h(X, Y)=g(X, Y) H$ for all $X, Y \in \Gamma(T M)$.

Let $M$ be any submanifold of a g.c.K. manifold $(\bar{M}, J, \omega, g)$. Then the Gauss and Weingarten formulas with respect to $\tilde{\nabla}$ are given by

$$
\begin{gathered}
\tilde{\nabla}_{X} Y=\hat{\nabla}_{X} Y+\tilde{h}(X, Y), \\
\tilde{\nabla}_{X} Z=-\tilde{A}_{Z} X+\tilde{\nabla}_{X}^{\perp} Z,
\end{gathered}
$$

for $X, Y \in \Gamma(T M)$ and $Z \in \Gamma\left(T^{\perp} M\right)$. Thus, using (2.7), (2.9) (2.13), we have the following relations.

$$
\begin{gathered}
\hat{\nabla}_{X} Y=\nabla_{X} Y-\frac{1}{2}\left\{\omega(X) Y+\omega(Y) X-g(X, Y) B^{T}\right\}, \\
\tilde{h}(X, Y)=h(X, Y)+\frac{1}{2} g(X, Y) B^{N}, \\
\tilde{A}_{Z} X=A_{Z} X+\frac{1}{2} \omega(Z) X,
\end{gathered}
$$

where $X, Y \in \Gamma(T M)$ and $Z \in \Gamma\left(T^{\perp} M\right)$, where $B^{T}$ and $B^{N}$ are the tangential and the normal part of $B$, respectively. 


\section{Semi-slant submanifolds of a g.c.K. manifold}

In this section, we recall some fundamental properties of semi-slant submanifolds of a g.c.K. manifold given in [23] and give some auxiliary results to prove our main theorem.

Let $(\bar{M}, J, g)$ be an almost Hermitian manifold and let $M$ be a Riemannian manifold isometrically immersed in $\bar{M}$. A distribution $\mathcal{D}$ on $M$ is called a slant distribution if for $V \in \mathcal{D}_{p}$, the angle $\theta$ between $J V$ and $\mathcal{D}_{p}$ is constant, i.e., independent of $p \in M$ and $V \in \mathcal{D}_{p}$. The constant angle $\theta$ is called the slant angle of the slant distribution $\mathcal{D}$. We know that holomorphic and totally real distributions on $M$ are slant distributions with $\theta=0$ and $\theta=\frac{\pi}{2}$, respectively. A slant distribution is called proper if it is neither holomorphic nor totally real. A submanifold $M$ of $\bar{M}$ is said to be a slant submanifold [6] if the tangent bundle $T M$ of $M$ is slant. For examples and more details, (see [6]).

A semi-slant submanifold $M[17]$ of a g.c.K. manifold $(\bar{M}, J, \omega, g)$ is a submanifold such that its tangent bundle $T M$ admits two orthogonal complementary holomorphic distribution $\mathcal{D}^{T}$ and slant distribution $\mathcal{D}^{\theta}$, i.e., we have

$$
T M=\mathcal{D}^{T} \oplus \mathcal{D}^{\theta} .
$$

We say that a semi-slant submanifold $M$ is proper if $\operatorname{dim}\left(\mathcal{D}^{T}\right) \neq\{0\}$ and $\theta \neq 0, \frac{\pi}{2}$.

For any $X \in T M$ we write

$$
J X=P X+F X
$$

where $P X$ is the tangential part of $J X$, and $F X$ is the normal part of $J X$. Then the normal bundle $T^{\perp} M$ of $M$ is decomposed as

$$
T^{\perp} M=F \mathcal{D}^{\theta} \oplus \overline{\mathcal{D}}
$$

where $\overline{\mathcal{D}}$ is the orthogonal complementary distribution of $F \mathcal{D}^{\theta}$ in $T^{\perp} M$ and it is invariant subbundle of $T^{\perp} M$ with respect to $J$. For a semi-slant submanifold [21], we have

$$
\begin{gathered}
P^{2} V=-\cos ^{2} \theta V, \\
g(P U, P V)=\cos ^{2} \theta g(U, V) \quad \text { and } \quad g(F U, F V)=\sin ^{2} \theta g(U, V)
\end{gathered}
$$

for $U, V \in \Gamma\left(\mathcal{D}^{\theta}\right)$.

Lemma 3.1. Let $M$ be a semi-slant submanifold of a g.c.K. manifold $(\bar{M}, J, \omega, g)$. Then we have

$$
\begin{aligned}
g\left(\nabla_{X} Y, V\right)= & \csc ^{2} \theta\left\{g\left(A_{F V} J Y-A_{F P V} Y, X\right)+\frac{1}{2} \omega(F V) g(J Y, X)\right. \\
& \left.-\frac{1}{2} \omega(F P V) g(X, Y)\right\}-\frac{1}{2} \omega(V) g(X, Y),
\end{aligned}
$$

for any $X, Y \in \Gamma\left(\mathcal{D}^{T}\right)$ and $V \in \Gamma\left(\mathcal{D}^{\theta}\right)$.

Proof. Let $X, Y \in \Gamma\left(\mathcal{D}^{T}\right)$ and $V \in \Gamma\left(\mathcal{D}^{\theta}\right)$, since $\left(\bar{M}, J, \omega, \tilde{g}=e^{-\sigma} g\right)$ is a Kaehler manifold, using (2.8), (2.12), (2.13) and (3.2), we have

$$
\begin{aligned}
\tilde{g}\left(\hat{\nabla}_{X} Y, V\right) & =\tilde{g}\left(\tilde{\nabla}_{X} Y, V\right)=\tilde{g}\left(\tilde{\nabla}{ }_{X} J Y, J V\right) \\
& =\tilde{g}\left(\tilde{\nabla}_{X} J Y, P V\right)+\tilde{g}\left(\tilde{\nabla}_{X} J Y, F V\right) \\
& =-\tilde{g}\left(\tilde{\nabla}_{X} Y, J P V\right)+\tilde{g}\left(\tilde{A}_{F V} X, J Y\right) \\
& =-\tilde{g}\left(\tilde{\nabla}_{X} Y, P^{2} V\right)-\tilde{g}\left(\tilde{\nabla}_{X} Y, F P V\right)+\tilde{g}\left(\tilde{A}_{F V} X, J Y\right) \\
& =\cos ^{2} \theta \tilde{g}\left(\hat{\nabla}_{X} Y, V\right)+\tilde{g}\left(\tilde{A}_{F V} J Y, X\right)-\tilde{g}\left(\tilde{A}_{F P V} Y, X\right) .
\end{aligned}
$$

Hence, it follows that

$$
\tilde{g}\left(\hat{\nabla}_{X} Y, V\right)=\csc ^{2} \theta \tilde{g}\left(\tilde{A}_{F V} J Y, X\right)-\tilde{g}\left(\tilde{A}_{F P V} Y, X\right) .
$$

Now, by using (2.6), (2.14) and (2.16), we derive the conclusion. 
Using the above Lemma, we have the following result.

Theorem 3.2. Let $M$ be a proper semi-slant submanifold of a g.c.K. manifold $(\bar{M}, J, \omega, g)$. Then the holomorphic distribution $\mathcal{D}^{T}$ is totally geodesic if and only if

$$
g\left(A_{F V} J Y-A_{F P V} Y, X\right)=\frac{1}{2}\left\{\left(\sin ^{2} \theta \omega(V)+\omega(F P V)\right) g(Y, X)-\omega(F V) g(J Y, X)\right\}
$$

for $X, Y \in \Gamma\left(\mathcal{D}^{T}\right)$ and $V \in \Gamma\left(\mathcal{D}^{\theta}\right)$.

Lemma 3.3. Let $M$ be a semi-slant submanifold of a g.c.K. manifold $(\bar{M}, J, \omega, g)$. Then we have

$$
g\left(\nabla_{U} V, X\right)=-\csc ^{2} \theta g\left(A_{F V} J X-A_{F P V} X, U\right)-\frac{1}{2} \omega(X) g(U, V)
$$

for any $X \in \Gamma\left(\mathcal{D}^{T}\right)$ and $U, V \in \Gamma\left(\mathcal{D}^{\theta}\right)$.

Proof. Let $X \in \Gamma\left(\mathcal{D}^{T}\right)$ and $U, V \in \Gamma\left(\mathcal{D}^{\theta}\right)$, since $\left(\bar{M}, J, \omega, \tilde{g}=e^{-\sigma} g\right)$ is a Kaehler manifold, using (2.8), (2.12), (2.13) and (3.2), we have

$$
\begin{aligned}
\tilde{g}\left(\hat{\nabla}_{U} V, X\right) & =\tilde{g}\left(\tilde{\nabla}_{U} V, X\right)=\tilde{g}\left(\tilde{\nabla} \tilde{\nabla}_{U} J V, J X\right) \\
& =\tilde{g}\left(\tilde{\nabla}_{U} P V, J X\right)+\tilde{g}\left(\tilde{\nabla}_{U} F V, J X\right) \\
& =-\tilde{g}\left(\tilde{\nabla}_{U} J P V, X\right)-\tilde{g}\left(\tilde{A}_{F V} J X, U\right) \\
& =-\tilde{g}\left(\tilde{\nabla}_{U} P^{2} V, X\right)-\tilde{g}\left(\tilde{\nabla}_{U} F P V, X\right)-\tilde{g}\left(\tilde{A}_{F V} J X, U\right) \\
& =\cos ^{2} \theta \tilde{g}\left(\hat{\nabla}_{U} V, X\right)+\tilde{g}\left(\tilde{A}_{F P V} X, U\right)-\tilde{g}\left(\tilde{A}_{F V} J X, U\right) .
\end{aligned}
$$

Hence, it follows that

$$
\tilde{g}\left(\hat{\nabla}_{U} V, X\right)=-\csc ^{2} \theta \tilde{g}\left(\tilde{A}_{F V} J X-\tilde{A}_{F P V} X, U\right) .
$$

Now, by using (2.6), (2.14) and (2.16), we derive the conclusion.

By using (3.7), we obtain the following result.

Theorem 3.4. Let $M$ be a proper semi-slant submanifold of a g.c.K. manifold $(\bar{M}, J, \omega, g)$. Then the slant distribution $\mathcal{D}^{\theta}$ is integrable if and only if

$$
g\left(A_{F V} J X-A_{F P V} X, U\right)=g\left(A_{F U} J X-A_{F P U} X, V\right)
$$

for $X \in \Gamma\left(\mathcal{D}^{T}\right)$ and $U, V \in \Gamma\left(\mathcal{D}^{\theta}\right)$.

Now, we give totally geodesicness condition of $\mathcal{D}^{\theta}$ and integrability condition of $\mathcal{D}^{T}$, respectively.

Theorem 3.5. Let $M$ be a proper semi-slant submanifold of a g.c.K. manifold $(\bar{M}, J, \omega, g)$. Then the slant distribution $\mathcal{D}^{\theta}$ is totally geodesic if and only if

$$
g\left(A_{F V} J X-A_{F P V} X, U\right)=-\frac{1}{2} \sin ^{2} \theta \omega(X) g(U, V)
$$

for $X \in \Gamma\left(\mathcal{D}^{T}\right)$ and $U, V \in \Gamma\left(\mathcal{D}^{\theta}\right)$.

Proof. Let $M$ be a proper semi-slant submanifold of a g.c.K. manifold $(\bar{M}, J, \omega, g)$. The slant distribution $\mathcal{D}^{\theta}$ is totally geodesic if and only if $g\left(\nabla_{U} V, X\right)=0$ for any $U, V \in \Gamma\left(\mathcal{D}^{\theta}\right)$ and $X \in \Gamma\left(\mathcal{D}^{T}\right)$. From (3.7), $g\left(\nabla_{U} V, X\right)=0$ if and only if (3.9) holds.

Remark 3.6. In [23], the authors gave a different condition for the totally geodesicness of the slant distribution $\mathcal{D}^{\theta}$.

Theorem 3.7. Let $M$ be a proper semi-slant submanifold of a g.c.K. manifold $(\bar{M}, J, \omega, g)$. Then the holomorphic distribution $\mathcal{D}^{T}$ is integrable if and only if

$$
g\left(A_{F V} J Y-A_{F P V} Y, X\right)+\omega(F V) g(J Y, X)=g\left(A_{F V} J X-A_{F P V} X, Y\right)
$$

for $X, Y \in \Gamma\left(\mathcal{D}^{T}\right)$ and $V \in \Gamma\left(\mathcal{D}^{\theta}\right)$. 
Proof. Let $M$ be a proper semi-slant submanifold of a g.c.K. manifold $(\bar{M}, J, \omega, g)$. The holomorphic distribution $\mathcal{D}^{T}$ is integrable if and only if $g([X, Y], V)=0$ for any $X, Y \in$ $\Gamma\left(\mathcal{D}^{T}\right)$ and $V \in \Gamma\left(\mathcal{D}^{\theta}\right)$. From (3.6), $g([X, Y], V)=0$ if and only if (3.10) holds.

Remark 3.8. In [23], the authors gave a different condition for the integrability of the holomorphic distribution $\mathcal{D}^{T}$.

Remark 3.9. Throughout this paper, for a semi-slant submanifold $M$ of a g.c.K. manifold $(\bar{M}, J, \omega, g)$, we write $B=B^{T}+B^{\theta}$, where $B^{T}$ is the tangent part of $B$ to $\mathcal{D}^{T}$ and $B^{\theta}$ is the tangent part of $B$ to $\mathcal{D}^{\theta}$.

\section{Conformal-twisted product semi-slant submanifolds of a g.c.K. mani- fold}

In this section, we study conformal-twisted product semi-slant submanifolds in the form ${ }_{f} M^{T} \times_{b} M^{\theta}$ with conformal factor $f$ defined on $M^{T}$ and twisting function $b$ of a g.c.K. manifold, where $M^{T}$ is a holomorphic and $M^{\theta}$ is a slant submanifold of the g.c.K. manifold. We first give an (non-trivial) example of such a submanifold in a g.c.K. manifold.

Example 4.1. Let $\left(z_{1}, \ldots, z_{6}\right)$ be natural coordinates of the six-dimensional Euclidean space $R^{6}$ and let $\bar{R}^{6}=\left\{\left(z_{1}, \ldots, z_{6}\right) \in R^{6}: z_{1}, z_{2}, z_{5} \neq 0\right\}$. Then $\left(\bar{R}^{6}, J, g_{0}\right)$ is a Kaehler manifold with usual Kaehler structure $\left(J, g_{0}\right)$. Now, we consider the Riemannian metric $g=e^{\sigma} g_{0}$ conformal to Kaehler metric $g_{0}$ on $\bar{R}^{6}$, where $e^{\sigma}=\left(z_{1} z_{2}\right)^{2}$. Then $\left(\bar{R}^{6}, J, g\right)$ is clearly a g.c.K. manifold. Let $M$ be a submanifold given by

$$
z_{1}=x, z_{2}=y, z_{3}=u+v, z_{4}=-u+v, z_{5}=u, z_{6}=0,
$$

where $x, y, u \neq 0$ and $v>1$. Then, the local frame field of the tangent bundle $T M$ of $M$ is given by

$$
X=\partial_{1}, \quad Y=\partial_{2}, \quad U=\frac{1}{\sqrt{3}}\left\{\partial_{3}-\partial_{4}+\partial_{5}\right\}, \quad V=\frac{1}{\sqrt{2}}\left\{\partial_{3}+\partial_{4}\right\},
$$

where $\partial_{i}=\frac{\partial}{\partial z_{i}}$ for $i \in\{1,2, \ldots, 6\}$. Then $\mathcal{D}^{T}=\operatorname{span}\{X, Y\}$ is a holomorphic and $\mathcal{D}^{\theta}=$ $\operatorname{span}\{U, V\}$ is a (proper) slant distribution with the slant angle $\theta=\cos ^{-1}\left(\frac{2}{\sqrt{6}}\right)$. Thus, $M$ is a proper semi-slant submanifold of $\left(\bar{R}^{6}, J, g\right)$. We easily observe both $\mathcal{D}^{T}$ and $\mathcal{D}^{\theta}$ are integrable. Let denote the integral submanifolds of $\mathcal{D}^{T}$ and $\mathcal{D}^{\theta}$ by $M^{T}$ and $M^{\theta}$, respectively. Let $g_{T}$ and $g_{\theta}$ be the induced metrics on $M^{T}$ and $M^{\theta}$ with respect to the Kaehler metric $g_{0}$, respectively. We choose the conformal metric $\bar{g}_{\theta}=\frac{1}{v^{2}} g_{\theta}$ on $M^{\theta}$. Since $x=z_{1}$ and $y=z_{2}$ on $M$, the induced metric of $M$ from the conformal Kaehler metric $g$ is

$$
\begin{aligned}
d s^{2} & =(x y)^{2}\left(d x^{2}+d y^{2}\right)+(x y)^{2}\left(d u^{2}+d v^{2}\right) \\
& =x^{2} y^{2} g_{T}+x^{2} y^{2} g_{\theta} \\
& =x^{2} y^{2} g_{T}+x^{2} y^{2} v^{2} \bar{g}_{\theta} .
\end{aligned}
$$

Thus, $M$ is a conformal-twisted product of $\left(M^{T}, g_{T}\right)$ and $\left(M^{\theta}, \bar{g}_{\theta}\right)$. So, $M$ is a non-trivial conformal-twisted product proper semi-slant submanifold in the g.c.K. manifold $\left(\bar{R}^{6}, J, g\right)$ in the form ${ }_{f} M^{T} \times_{b} M^{\theta}$ with conformal factor $f=x y$ and twisting function $b=x y v$. Moreover, the Lee form of $\left(\bar{R}^{6}, J, g\right)$ is $\omega=2\left\{\frac{1}{x} d x+\frac{1}{y} d y\right\}$. Consequently, the Lee vector field is

$$
B=\frac{2}{(x y)^{2}}\left\{\frac{1}{x} \frac{\partial}{\partial x}+\frac{1}{y} \frac{\partial}{\partial y}\right\}
$$

which is tangent to $M^{T}$. 
Lemma 4.2. Let $M={ }_{f} M^{T} \times{ }_{b} M^{\theta}$ be a conformal-twisted product semi-slant submanifold of a g.c.K. manifold $(\bar{M}, J, \omega, g)$. Then, for all $V \in \mathcal{L}\left(M^{\theta}\right)$, we have

$$
\omega(V)=0 \text {. }
$$

Proof. Let $M={ }_{f} M^{T} \times{ }_{b} M^{\theta}$ be a conformal-twisted semi-slant submanifold of a g.c.K. manifold $(\bar{M}, J, \omega, g)$ and $V \in \mathcal{L}\left(M^{\theta}\right)$ and $X, Y \in \mathcal{L}\left(M^{T}\right)$. Then using the exterior differentiation formula (see, [29], p. 17), we have

$$
\begin{aligned}
3 d \Omega(V, X, Y) & =V \Omega(X, Y)+X \Omega(Y, V)+Y \Omega(V, X) \\
& -\Omega([V, X], Y)-\Omega([X, Y], V)-\Omega([Y, V], X) \\
& =V g(X, J Y)-X g(J Y, V)+Y g(V, J X) \\
& -g([V, X], J Y)+g(J[X, Y], V)-g([Y, V], J X) .
\end{aligned}
$$

Here, we know $g(J Y, V)=g(V, J X)=0$, since $M$ is a semi-slant submanifold. Also, by (2.3), we have $[V, X]=[Y, V]=0$ and by $(2.2)$, we have $[X, Y]=\nabla_{X}^{T} Y-\nabla_{Y}^{T} X$. So $J[X, Y] \in \mathcal{L}\left(M^{T}\right)$. Thus, we obtain

$$
\begin{aligned}
3 d \Omega(V, X, Y) & =V g(X, J Y) \\
& =g\left(\nabla_{V} X, J Y\right)+g\left(\nabla_{V} Y, J X\right) .
\end{aligned}
$$

Again, using (2.3), we find

$$
3 d \Omega(V, X, Y)=X(\ln b) g(V, J Y)+Y(\ln b) g(V, J X)=0 .
$$

So, $d \Omega(V, X, Y)=0$. On the other hand, from (2.5) we have

$$
\begin{aligned}
d \Omega(V, X, Y) & =\omega \wedge \Omega(V, X, Y) \\
& =\omega(V) \Omega(X, Y)+\omega(X) \Omega(Y, V)+\omega(Y) \Omega(V, X) \\
& =\omega(V) g(X, J Y) .
\end{aligned}
$$

Since $g$ is non-degenerate, it follows that $\omega(V)=0$.

Lemma 4.3. Let $M={ }_{f} M^{T} \times{ }_{b} M^{\theta}$ be a conformal-twisted product semi-slant submanifold of a g.c.K. manifold $(\bar{M}, J, \omega, g)$. Then, for all $X \in \mathcal{L}\left(M^{T}\right)$, we have

$$
\omega(X)=\frac{2}{3} X(\ln b)
$$

Proof. Let $M={ }_{f} M^{T} \times{ }_{b} M^{\theta}$ be a conformal-twisted product semi-slant submanifold of a g.c.K. manifold $(\bar{M}, J, \omega, g)$ and $U, V \in \mathcal{L}\left(M^{\theta}\right)$ and $X \in \mathcal{L}\left(M^{T}\right)$. Then, we have

$$
\begin{aligned}
3 d \Omega(X, U, V) & =X \Omega(U, V)+U \Omega(V, X)+V \Omega(X, U) \\
& -\Omega([X, U], V)-\Omega([U, V], X)-\Omega([V, X], U) \\
& =X g(U, P V),
\end{aligned}
$$

since $[X, V]=[X, U]=0$ from $(2.3)$ and $[U, V]=\nabla_{U}^{\theta} V-\nabla_{V}^{\theta} U \in \mathcal{L}\left(M^{\theta}\right)$ from (2.4). After some calculation in view of $(2.3)$, we obtain

$$
3 d \Omega(X, U, V)=2 X(\ln b) g(U, P V) .
$$

On the other hand, we have

$$
\begin{aligned}
d \Omega(X, U, V) & =\omega \wedge \Omega(X, U, V) \\
& =\omega(X) \Omega(U, V)+\omega(U) \Omega(V, X)+\omega(V) \Omega(X, U) \\
& =\omega(X) g(U, P V) .
\end{aligned}
$$

from (2.5). Namely,

$$
d \Omega(X, U, V)=\omega(X) g(U, P V) .
$$

Thus, the assertion follows from (4.3) and (4.4).

Remark 4.4. In Kaehlerian case, we have $\omega(X)=\frac{2}{3} X(\ln b)=0$, since $\omega=0$. It follows that twisting function $b$ depends only on the points of $M^{\theta}$. Then, we have $g_{M}=$ $f^{2} g_{T} \oplus b^{2} g_{\theta}$. Then, $M$ is a locally direct product of $\left(M_{T}, g_{1}\right)$ and $\left(M_{\theta}, g_{2}\right)$, where $g_{1}=f^{2} g_{T}$ and $g_{2}=b^{2} g_{\theta}$. 
Theorem 4.5. Let $M={ }_{f} M^{T} \times{ }_{b} M^{\theta}$ be a conformal-twisted product semi-slant submanifold of a g.c.K. manifold $(\bar{M}, J, \omega, g)$. Then $M$ is a locally direct product manifold if and only if the Lee vector field $B$ is normal to $M$.

Proof. Let $M$ be a direct product semi-slant submanifold of and $\left(M^{T}, g_{T}\right)$ and $\left(M^{\theta}, \bar{g}_{\theta}\right)$, where $\bar{g}_{\theta}=c g_{\theta}$ for some constant $c$. Then the induced metric tensor $g_{M}$ of $M$ has the form $g_{M}=g_{T} \oplus \bar{g}_{\theta}$, where $g_{T}$ and $g_{\theta}$ are the induced metrics on $M^{T}$ and $M^{\theta}$, respectively. By Lemma 4.3, for any $X \in \mathcal{L}\left(M^{T}\right)$, we have $\frac{2}{3} X(\ln b)=\omega(X)=0$, since $b$ is a constant function. With the help of $(2.6)$, it follows that $\omega(X)=g(B, X)=0$. So $B$ is normal to $M^{T}$. By this fact together with (4.1), we deduce that $\omega(V)=g(B, V)=0$, for $V \in \mathcal{L}\left(M^{\theta}\right)$. Also, $B$ is normal to $M_{\theta}$. Thus $B$ is normal to $M$.

Conversely, let $B$ be normal to $M$. Then for any $X \in \mathcal{L}\left(M^{T}\right),(4.2)$ implies $\frac{2}{3} X(\ln b)=$ $\omega(X)=g(B, X)=0$. Which says us the twisting function depends only on the points $M_{\theta}$. Then the induced metric tensor $g_{M}$ of $M$ has the form $g_{M}=f^{2} g_{T} \oplus b^{2} g_{T}$, where $f$ and $g$ depend only on the points $M_{T}$ and $M_{\theta}$, respectively. Hence, we conclude that $M$ is a locally direct product of $\left(M_{T}, g_{1}\right)$ and $\left(M_{\theta}, g_{2}\right)$, where $g_{1}=f^{2} g_{T}$ and $g_{2}=b^{2} g_{\theta}$.

Remark 4.6 ([18, Proposition 3-b]). Let $g$ be a pseudo Riemannian metric on the manifold $M_{1} \times M_{2}$ and assume that the canonical foliations $\mathcal{D}_{1}$ and $\mathcal{D}_{2}$ intersect perpendicularly everywhere. Then $g$ is the metric of a twisted product ${ }_{1} M_{1} \times{ }_{f_{1}} M_{2}$ if and only if $\mathcal{D}_{1}$ is a totally geodesic and $\mathcal{D}_{2}$ is a totally umbilic foliation.

Remark 4.7. Actually, twisted products and conformal-twisted products can be expressed in terms of each other. Indeed, let $\left(M_{1} \times{ }_{b} M_{2}, g\right)$ be a twisted product manifold, where $g=g_{1} \oplus b^{2} g_{2}$ with twisting function $b$. Choosing conformal metric $g_{1}=f^{2} \bar{g}_{1}$, where the positive smooth function $f$ depends only on the points of $M_{1}$, the metric $g$ is of the form $g=f^{2} \bar{g}_{1} \oplus b^{2} g_{2}$. So, $\left(M_{1} \times_{b} M_{2}, g\right)$ is a conformal-twisted product of $\left(M_{1}, \bar{g}_{1}\right)$ and $\left(M_{2}, g_{2}\right)$. On the other hand, let $\left({ }_{f} M_{1} \times_{b} M_{2}, g\right)$ be a conformal-twisted product manifold with conformal factor $f$ and twisting function $b$. Choosing conformal metric $\bar{g}_{1}=f^{2} g_{1}$, the metric $g$ is of the form $g=\bar{g}_{1} \oplus b^{2} g_{2}$. Thus, $\left({ }_{f} M_{1} \times{ }_{b} M_{2}, g\right)$ is a twisted product of $\left(M_{1}, \bar{g}_{1}\right)$ and $\left(M_{2}, g_{2}\right)$.

Now, we are ready to prove main theorem.

Theorem 4.8. Let $M$ be a proper semi-slant submanifold of a g.c.K. manifold $(\bar{M}, J, \omega, g)$. Then $M$ is a locally conformal-twisted product submanifold of type ${ }_{f} M^{T} \times_{b} M^{\theta}$ if and only if for any $X \in \Gamma\left(\mathcal{D}^{T}\right)$ and $V \in \Gamma\left(\mathcal{D}^{\theta}\right)$, we have

$$
\begin{gathered}
\omega(V)=0, \\
\omega(X)=X(\mu), \\
A_{F V} J X-A_{F P V} X=\frac{1}{2}\{\omega(F P V) X-\omega(F V) J X\} \\
+\sin ^{2} \theta \omega(X) V
\end{gathered}
$$

for some function $\mu$.

Proof. Let $M$ be a conformal-twisted product submanifold of the g.c.K. manifold ( $\bar{M}, J, \omega, g)$ of type ${ }_{f} M^{T} \times_{b} M^{\theta}$. The equations (4.5) is already obtained in Lemma 4.2. On the other hand, we have $\omega(X)=\frac{2}{3} X(\ln b)$ from Lemma 4.3. Thus, we get (4.6) for $\mu=\frac{2}{3} \ln b$. Now, for any $X \in \mathcal{L}\left(M^{T}\right)$ and $V \in \mathcal{L}\left(M^{\theta}\right)$, we write

$$
A_{F V} J X-A_{F P V} X=\left(A_{F V} J X-A_{F P V} X\right)^{T}+\left(A_{F V} J X-A_{F P V} X\right)^{\theta}
$$


where $\left(A_{F V} J X-A_{F P V} X\right)^{T}$ is the tangent part of $A_{F V} J X-A_{F P V} X$ to $M^{T}$ and $\left(A_{F V} J X-A_{F P V} X\right)^{\theta}$ is the tangent part of $A_{F V} J X-A_{F P V} X$ to $M^{\theta}$. Hence, for any $Y \in \mathcal{L}\left(M^{T}\right)$, using (2.2) and (4.1), we have

$$
g\left(A_{F V} J X-A_{F P V} X, Y\right)=\frac{1}{2}\{\omega(F P V) g(X, Y)-\omega(F V) g(J X, Y)\}
$$

from (3.6). Hence, it follows that

$$
\left(A_{F V} J X-A_{F P V} X\right)^{T}=\frac{1}{2}\{\omega(F P V) X-\omega(F V) J X\} .
$$

Similarly, for any $U \in \mathcal{L}\left(M^{\theta}\right)$, using (2.3) and (4.2), we have

$$
g\left(A_{F V} J X-A_{F P V} X, U\right)=\sin ^{2} \theta X\left(\frac{2}{3} \ln b\right) g(U, V)
$$

from (3.7). Since $U \in \mathcal{L}\left(M^{\theta}\right)$ is arbitrary and the metric $g$ is Riemann, it follows that

$$
\left(A_{F V} J X-A_{F P V} X\right)^{\theta}=\sin ^{2} \theta \omega(X) V .
$$

By using (4.8) (4.10), we get (4.7).

Conversely, suppose that $M$ is a semi-slant submanifold of a g.c.K. manifold $(\bar{M}, J, \omega, g)$ such that (4.5) (4.7) hold. Then, for any $X \in \Gamma\left(\mathcal{D}^{T}\right)$ and $U, V \in \Gamma\left(\mathcal{D}^{\theta}\right)$, using (4.5) and (4.7), we obtain (3.6). Thus, by Theorem 3.2, the holomorphic distribution $\mathcal{D}^{T}$ is totally geodesic. On the other hand, using (4.7), we deduce (3.8). Thus, by Theorem 3.4, the slant distribution $\mathcal{D}^{\theta}$ is integrable. Let $M^{T}$ and $M^{\theta}$ be the integral manifolds of $\mathcal{D}^{T}$ and $\mathcal{D}^{\theta}$, respectively and let denote by $h^{T}$ and $h^{\theta}$ the second fundamental forms of $M^{T}$ and $M^{\theta}$ in $M$, respectively. Then, for any $X, Y \in \Gamma\left(\mathcal{D}^{T}\right)$ and $V \in \Gamma\left(\mathcal{D}^{\theta}\right)$, using (2.9), we have

$$
g\left(h^{T}(X, Y), V\right)=g\left(\nabla_{X} Y, V\right) .
$$

Here, if we use (4.5) and (4.7), we deduce that

$$
g\left(h^{T}(X, Y), V\right)=0
$$

from (3.6). Which means that $M^{T}$ is totally geodesic in $M$. On the other hand, for any $X \in \Gamma\left(\mathcal{D}^{T}\right)$ and $U, V \in \Gamma\left(\mathcal{D}^{\theta}\right)$, using (2.9), we have

$$
g\left(h^{\theta}(U, V), X\right)=g\left(\nabla_{U} V, X\right) .
$$

Here, using (4.6) and (4.7), we obtain

$$
g\left(h^{\theta}(U, V), X\right)=-X(\ln b) g(U, V)
$$

from (3.7). After some calculation, we obtain

$$
g\left(h^{\theta}(U, V), X\right)=-g(g(U, V) \nabla(\ln b), X) .
$$

Hence, we conclude that

$$
h^{\theta}(U, V)=g(U, V)(-\nabla(\ln b)) .
$$

It means that $M^{\theta}$ is totally umbilic in $M$ with parallel mean curvature vector field $-\nabla(\ln b)$. Hence, by Remark 4.6 and Remark 4.7, we know that $M$ is conformal-twisted product ${ }_{f} M^{T} \times_{b} M^{\theta}$ of a holomorphic submanifold $M^{T}$ and a slant submanifold $M^{\theta}$ of $M$, where $M^{T}$ is a leaf of $\mathcal{D}^{T}$ and $M^{\theta}$ is leaf of $\mathcal{D}^{\theta}$ and $b$ is a certain twisting function. 


\section{An inequality for conformal-twisted product semi-slant submanifolds in the form ${ }_{f} M^{T} \times{ }_{b} M^{\theta}$}

In this section, we shall establish an inequality for the squared norm of the second fundamental form of a conformal-twisted product semi-slant submanifold in the form ${ }_{f} M^{T} \times_{b} M^{\theta}$, where $M^{T}$ is a holomorphic and $M^{\theta}$ is a slant submanifold of a g.c.K. manifold $(\bar{M}, J, \omega, g)$.

Lemma 5.1. Let $M={ }_{f} M^{T} \times_{b} M^{\theta}$ be a conformal-twisted product semi-slant submanifold of a g.c.K. manifold $(\bar{M}, J, \omega, g)$ and $h$ be the second fundamental form of $M$ in $\bar{M}$. Then we have

$$
\begin{gathered}
g(h(X, Y), F V)=-\frac{1}{2} g(X, Y) \omega(F V), \\
g(h(X, U), F V)=-\omega(J X) g(U, V)-\omega(X) g(U, P V),
\end{gathered}
$$

where $X, Y \in \mathcal{L}\left(M^{T}\right)$ and $U, V \in \mathcal{L}\left(M^{\theta}\right)$.

Proof. Let $M={ }_{f} M^{T} \times_{b} M^{\theta}$ be a conformal-twisted product semi-slant submanifold of a g.c.K. manifold $(\bar{M}, J, \omega, g)$ and let $X, Y \in \mathcal{L}\left(M^{T}\right)$ and $V \in \mathcal{L}\left(M^{\theta}\right)$, since $(\bar{M}, J, \omega, \tilde{g}=$ $\left.e^{-\sigma} g\right)$ is a Kaehler manifold, using (2.12), (3.2) and (2.8), we have

$$
\begin{aligned}
\tilde{g}(\tilde{h}(X, Y), F V) & =\tilde{g}\left(\tilde{\nabla}_{X} Y, F V\right) \\
& =\tilde{g}\left(\tilde{\nabla}_{X} Y, J V\right)-\tilde{g}\left(\tilde{\nabla}_{X} Y, P V\right) \\
& =-\tilde{g}\left(\tilde{\nabla}_{X} J Y, V\right)-\tilde{g}\left(\hat{\nabla}_{X} Y, P V\right) \\
& =-\tilde{g}\left(\hat{\nabla}_{X} J Y, V\right)-\tilde{g}\left(\hat{\nabla}_{X} Y, P V\right) .
\end{aligned}
$$

Now, using (2.14), (2.15) and (4.1), we get (5.1). Next, let $X, Y \in \mathcal{L}\left(M^{T}\right)$ and $V \in \mathcal{L}\left(M^{\theta}\right)$, since $\left(\bar{M}, J, \omega, \tilde{g}=e^{-\sigma} g\right)$ is a Kaehler manifold, using (2.12), (3.2) and (2.8), we have

$$
\begin{aligned}
\tilde{g}(\tilde{h}(X, U), F V) & =\tilde{g}\left(\tilde{\nabla}_{U} X, F V\right) \\
& =\tilde{g}\left(\tilde{\nabla}_{U} X, J V\right)-\tilde{g}\left(\tilde{\nabla}_{U} X, P V\right) \\
& =-\tilde{g}\left(\tilde{\nabla}_{U} J X, V\right)-\tilde{g}\left(\tilde{\nabla}_{U} X, P V\right) \\
& =-\tilde{g}\left(\hat{\nabla}_{U} J X, V\right)-\tilde{g}\left(\hat{\nabla}_{U} X, P V\right) .
\end{aligned}
$$

Now, using (2.3), (2.14), (2.15), (4.1) and (4.2), we get (5.2).

Let $M={ }_{f} M^{T} \times{ }_{b} M^{\theta}$ be a conformal-twisted product semi-slant submanifold of a g.c.K. manifold $(\bar{M}, J, \omega, g)$. We choose a canonical orthonormal basis $\left\{e_{1}, \ldots, e_{m_{1}}, \bar{e}_{1}, \ldots, \bar{e}_{m_{2}}, e_{1}^{*}, \ldots, e_{m_{2}}^{*}, \hat{e_{1}}, \ldots, \hat{e}_{l}\right\}$ of $\bar{M}$ such that $\left\{e_{1}, \ldots, e_{m_{1}}\right\}$ is an orthonormal basis of $\mathcal{D}^{T},\left\{\bar{e}_{1}, \ldots, \bar{e}_{m_{2}}\right\}$ is an orthonormal basis of $\mathcal{D}^{\theta},\left\{e_{1}^{*}, \ldots, e_{m_{2}}^{*}\right\}$ is an orthonormal basis of $F \mathcal{D}^{\theta}$ and $\left\{\hat{e_{1}}, \ldots, \hat{e_{l}}\right\}$ is an orthonormal basis of $\overline{\mathcal{D}}$. Here, $m_{1}=\operatorname{dim}\left(\mathcal{D}^{T}\right), m_{2}=$ $\operatorname{dim}\left(\mathcal{D}^{\theta}\right)$ and $l=\operatorname{dim}(\overline{\mathcal{D}})$.

Remark 5.2. Since $\mathcal{D}^{T}$ is a holomorphic distribution, $\left\{J e_{1}, \ldots, J e_{m_{1}}\right\}$ is also an orthonormal basis of $\mathcal{D}^{T}$. Moreover, by (3.5), we observe that $\left\{\bar{a}_{1}=\sec \theta P \bar{e}_{2}, \bar{a}_{2}=-\sec \theta P \bar{e}_{1}, \ldots, \bar{a}_{2 n_{2}-1}=\sec \theta P \bar{e}_{2 n_{2}}, \bar{a}_{2 n_{2}}=-\sec \theta P \bar{e}_{2 n_{2}-1}\right\}$ is also an orthonormal basis of $\mathcal{D}^{\theta}$ and $\left\{\csc \theta F \bar{e}_{1}, \ldots, \csc \theta F \bar{e}_{m_{2}}\right\}$ is also an orthonormal basis of $F \mathcal{D}^{\theta}$, where $\theta$ is the slant angle of $\mathcal{D}^{\theta}$ and $m_{2}=2 n_{2}=\operatorname{dim}\left(M^{\theta}\right)$.

Theorem 5.3. Let $M={ }_{f} M^{T} \times{ }_{b} M^{\theta}$ be a conformal-twisted product semi-slant submanifold of a g.c.K. manifold $(\bar{M}, J, \omega, g)$. Then we have

(i) The squared norm of the second fundamental form $h$ of $M$ satisfies

$$
\|h\|^{2} \geq \frac{1}{4} m_{1}\left\|B^{F D^{\theta}}\right\|^{2}+m_{2}\left(1+m_{2} \cot ^{2} \theta\right)\left\|B^{T}\right\|^{2},
$$

where $m_{1}=\operatorname{dim}\left(M^{T}\right), m_{2}=\operatorname{dim}\left(M^{\theta}\right)$ and $B^{F D^{\theta}}$ is tangential part of $B$ to $F \mathcal{D}^{\theta}$.

(ii) If the equality sign of (5.3) holds identically, then $M^{\theta}$ is also umbilic in the ambient manifold $\bar{M}$. 
Proof. The squared norm of the second fundamental form $h$ can be written as

$$
\|h\|^{2}=\left\|h\left(\mathcal{D}^{T}, \mathcal{D}^{T}\right)\right\|^{2}+\left\|h\left(\mathcal{D}^{T}, \mathcal{D}^{\theta}\right)\right\|^{2}+\left\|h\left(\mathcal{D}^{\theta}, \mathcal{D}^{\theta}\right)\right\|^{2} .
$$

In view of decomposition (3.3), which can be explicitly written as follows:

$$
\begin{aligned}
\|h\|^{2}= & \sum_{r, s=1}^{m_{1}} \sum_{i=1}^{m_{2}} g\left(h\left(e_{r}, e_{s}\right), e_{i}^{*}\right)^{2}+\sum_{i, j=1}^{m_{2}} \sum_{r=1}^{m_{1}} g\left(h\left(e_{r}, \bar{e}_{i}\right), e_{j}^{*}\right)^{2} \\
& +\sum_{r, s=1}^{m_{1}} \sum_{t=1}^{l} g\left(h\left(e_{r}, e_{s}\right), \hat{e}_{t}\right)^{2}+\sum_{r=1}^{m_{1}} \sum_{i=1}^{m_{2}} \sum_{t=1}^{l} g\left(h\left(e_{r}, \bar{e}_{i}\right), \hat{e}_{t}\right)^{2} \\
& +\left\|h\left(\mathcal{D}^{\theta}, \mathcal{D}^{\theta}\right)\right\|^{2} .
\end{aligned}
$$

Hence, we have

$$
\|h\|^{2} \geq \sum_{r, s=1}^{m_{1}} \sum_{i=1}^{m_{2}} g\left(h\left(e_{r}, e_{s}\right), e_{i}^{*}\right)^{2}+\sum_{i, j=1}^{m_{2}} \sum_{r=1}^{m_{1}} g\left(h\left(\bar{e}_{i}, e_{r}\right), e_{j}^{*}\right)^{2} .
$$

By Remark 5.2, we obtain

$$
\|h\|^{2} \geq \sum_{r, s=1}^{m_{1}} \sum_{i=1}^{m_{2}} g\left(h\left(e_{r}, e_{s}\right), e_{i}^{*}\right)^{2}+\sum_{i, j=1}^{m_{2}} \sum_{r=1}^{m_{1}} g\left(h\left(\bar{e}_{i}, e_{r}\right), \csc \theta F \bar{e}_{j}\right)^{2} .
$$

Now, using (5.1) and (5.2), we get

$$
\begin{aligned}
\|h\|^{2} \geq & \frac{1}{4} \sum_{r, s=1}^{m_{1}} \sum_{i=1}^{m_{2}} g^{2}\left(e_{r}, e_{s}\right) \omega^{2}\left(e_{i}^{*}\right) \\
& +\csc ^{2} \theta \sum_{i, j=1}^{m_{2}} \sum_{r=1}^{m_{1}}\left\{\omega^{2}\left(J e_{r}\right) g^{2}\left(\bar{e}_{i}, \bar{e}_{j}\right)+\omega^{2}\left(e_{r}\right) g^{2}\left(\bar{e}_{i}, P \bar{e}_{j}\right)\right\} \\
& +2 \csc ^{2} \theta \sum_{i, j=1}^{m_{2}} \sum_{r=1}^{m_{1}} \omega\left(J e_{r}\right) g\left(\bar{e}_{i}, \bar{e}_{j}\right) \omega\left(e_{r}\right) g\left(\bar{e}_{i}, P \bar{e}_{j}\right) .
\end{aligned}
$$

Using (2.6), we obtain

$$
\begin{aligned}
\|h\|^{2} \geq & \frac{1}{4} \sum_{r, s=1}^{m_{1}} \sum_{i=1}^{m_{2}} g^{2}\left(e_{r}, e_{s}\right) g^{2}\left(B, e_{i}^{*}\right) \\
& +\csc ^{2} \theta \sum_{i, j=1}^{m_{2}} \sum_{r=1}^{m_{1}}\left\{g^{2}\left(B, J e_{r}\right) g^{2}\left(\bar{e}_{i}, \bar{e}_{j}\right)+g^{2}\left(B, e_{r}\right) g^{2}\left(\bar{e}_{i}, P \bar{e}_{j}\right)\right\} \\
& +2 \csc ^{2} \theta \sum_{i, j=1}^{m_{2}} \sum_{r=1}^{m_{1}} g\left(B, J e_{r}\right) g\left(\bar{e}_{i}, \bar{e}_{j}\right) g\left(B, e_{r}\right) g\left(\bar{e}_{i}, P \bar{e}_{j}\right) .
\end{aligned}
$$

Here, the term

$$
\begin{aligned}
& \sum_{i, j=1}^{m_{2}} \sum_{r=1}^{m_{1}} g\left(B, J e_{r}\right) g\left(\bar{e}_{i}, \bar{e}_{j}\right) g\left(B, e_{r}\right) g\left(\bar{e}_{i}, P \bar{e}_{j}\right) \\
& =\sum_{i, j=1}^{m_{2}} \sum_{r=1}^{m_{1}} g\left(B, J e_{r}\right) g\left(B, e_{r}\right) g\left(\bar{e}_{i}, \bar{e}_{j}\right) g\left(\bar{e}_{i}, P \bar{e}_{j}\right) \\
& =-\sum_{i, j=1}^{m_{2}} \sum_{r=1}^{m_{1}} g\left(J B, e_{r}\right) g\left(B, e_{r}\right) g\left(\bar{e}_{i}, \bar{e}_{j}\right) g\left(\bar{e}_{i}, P \bar{e}_{j}\right) \\
& =-g\left(J B^{T}, B^{T}\right) \sum_{i, j=1}^{m_{2}} g\left(\bar{e}_{i}, \bar{e}_{j}\right) g\left(\bar{e}_{i}, P \bar{e}_{j}\right)=0,
\end{aligned}
$$


since $g\left(J B^{T}, B^{T}\right)=0$. Thus, we arrive at

$$
\begin{aligned}
\|h\|^{2} \geq & \frac{1}{4} \sum_{r, s=1}^{m_{1}} \sum_{i=1}^{m_{2}} g^{2}\left(e_{r}, e_{s}\right) g^{2}\left(B, e_{i}^{*}\right) \\
& +\csc ^{2} \theta \sum_{i, j=1}^{m_{2}} \sum_{r=1}^{m_{1}}\left\{g^{2}\left(B, J e_{r}\right) g^{2}\left(\bar{e}_{i}, \bar{e}_{j}\right)+g^{2}\left(B, e_{r}\right) g^{2}\left(\bar{e}_{i}, P \bar{e}_{j}\right)\right\} .
\end{aligned}
$$

Here, for $i, j \in\left\{1,2, \ldots, m_{2}\right\}$, we have

$$
g\left(\bar{e}_{i}, P \bar{e}_{j}\right)= \begin{cases}\cos \theta & \text { if } i \neq j, \\ 0 & \text { if } i=j,\end{cases}
$$

since $\mathcal{D}^{\theta}$ is a slant distribution with slant angle $\theta$.

Consequently, $\sum_{i, j=1}^{m_{2}} g^{2}\left(\bar{e}_{i}, P \bar{e}_{j}\right)=m_{2}\left(m_{2}-1\right) \cos ^{2} \theta$. Thus, by direct calculation, we obtain the following inequality,

$$
\|h\|^{2} \geq \frac{1}{4} m_{1}\left\|B^{F D^{\theta}}\right\|^{2}+\csc ^{2} \theta\left\{m_{2}\left\|B^{T}\right\|^{2}+m_{2}\left(m_{2}-1\right) \cos ^{2} \theta\left\|B^{T}\right\|^{2}\right\} .
$$

Rearrange the last inequality, we get the inequality (5.3). If the equality sign of (5.3) holds identically, then we have $h\left(\mathcal{D}^{\theta}, \mathcal{D}^{\theta}\right)=0$ from (5.4). Namely, $h$ vanishes on $\mathcal{D}^{\theta}$. Since $\mathcal{D}^{\theta}$ is a umbilic distribution on $M$, it follows that $M^{\theta}$ is umbilic in $\bar{M}$.

\section{Conformal-twisted product semi-slant submanifolds of a g.c.K. mani- fold in the form ${ }_{f} M^{\theta} \times_{b} M^{T}$}

In this section, we study conformal-twisted product semi-slant submanifolds in the form ${ }_{f} M^{\theta} \times{ }_{b} M^{T}$ with conformal factor $f$ defined on $M^{\theta}$ and twisting function $b$ of a g.c.K. manifold, where $M^{T}$ is a holomorphic and $M^{\theta}$ is a slant submanifold of the g.c.K. manifold. We first give an (non-trivial) example of such a submanifold in a g.c.K. manifold.

Example 6.1. Let $\left(z_{1}, \ldots, z_{6}\right)$ be natural coordinates of the six-dimensional Euclidean space $R^{6}$ and let $\bar{R}^{6}=\left\{\left(z_{1}, \ldots, z_{6}\right) \in R^{6}: z_{1}, z_{2}, z_{5} \neq 0\right\}$. Then $\left(\bar{R}^{6}, J, g_{0}\right)$ is a Kaehler manifold with usual Kaehler structure $\left(J, g_{0}\right)$. Now, we consider the Riemannian metric $g=e^{\sigma} g_{0}$ conformal to Kaehler metric $g_{0}$ on $\bar{R}^{6}$, where $e^{\sigma}=\left(\frac{z_{3}+z_{4}}{2}\right)^{2}$. Then $\left(\bar{R}^{6}, J, g\right)$ is clearly a g.c.K. manifold. Let $M$ be a submanifold given by

$$
z_{1}=x, z_{2}=y, z_{3}=u+v, z_{4}=-u+v, z_{5}=u, z_{6}=0,
$$

where $x>1$ and $y, u, v \neq 0$. Then, the local frame field of the tangent bundle $T M$ of $M$ is given by

$$
X=\partial_{1}, \quad Y=\partial_{2}, \quad U=\frac{1}{\sqrt{3}}\left\{\partial_{3}-\partial_{4}+\partial_{5}\right\}, V=\frac{1}{\sqrt{2}}\left\{\partial_{3}+\partial_{4}\right\},
$$

where $\partial_{i}=\frac{\partial}{\partial z_{i}}$ for $i \in\{1,2, \ldots, 6\}$. Then $\mathcal{D}^{T}=\operatorname{span}\{X, Y\}$ is a holomorphic and $\mathcal{D}^{\theta}=$ $\operatorname{span}\{U, V\}$ is a (proper) slant distribution with the slant angle $\theta=\cos ^{-1}\left(\frac{2}{\sqrt{6}}\right)$. Thus, $M$ is a proper semi-slant submanifold of $\left(\bar{R}^{6}, J, g\right)$. We easily observe both $\mathcal{D}^{T}$ and $\mathcal{D}^{\theta}$ are integrable. Let denote the integral submanifolds of $\mathcal{D}^{T}$ and $\mathcal{D}^{\theta}$ by $M^{T}$ and $M^{\theta}$, respectively. Let $g_{T}$ and $g_{\theta}$ be the induced metrics on $M^{T}$ and $M^{\theta}$ with respect to the Kaehler metric $g_{0}$, respectively. We choose the conformal metric $\bar{g}_{T}=\frac{1}{x^{2}} g_{T}$ on $M^{T}$. Since 
$v=\frac{z_{3}+z_{4}}{2}$ on $M$, the induced metric of $M$ from the conformal Kaehler metric $g$ is

$$
\begin{aligned}
d s^{2} & =v^{2}\left(d u^{2}+d v^{2}\right)+v^{2}\left(d x^{2}+d y^{2}\right) \\
& =v^{2} g_{\theta}+v^{2} g_{T} \\
& =v^{2} g_{\theta}+v^{2} x^{2} \bar{g}_{T} .
\end{aligned}
$$

Thus, $M$ is a conformal-twisted product of $\left(M^{T}, \bar{g}_{T}\right)$ and $\left(M^{\theta}, g_{\theta}\right)$. So, $M$ is a non-trivial conformal-twisted product proper semi-slant submanifold in the g.c.K. manifold $\left(\bar{R}^{6}, J, g\right)$ in the form $M={ }_{f} M^{\theta} \times_{b} M^{T}$ with conformal factor $f=v$ and twisting function $b=x v$. Moreover, the Lee form of $\left(\bar{R}^{6}, J, g\right)$ is $\omega=2\left\{\frac{1}{v} d v\right\}$. Consequently, the Lee vector field is

$$
B=\frac{2}{v^{2}}\left\{\frac{1}{v} \frac{\partial}{\partial v}\right\}
$$

which is tangent to $M^{\theta}$.

Lemma 6.2. Let $M={ }_{f} M^{\theta} \times{ }_{b} M^{T}$ be a conformal-twisted product semi-slant submanifold of a g.c.K. manifold $(\bar{M}, J, \omega, g)$. Then, for all $V \in \mathcal{L}\left(M^{\theta}\right)$, we have

$$
\omega(V)=\frac{2}{3} V(\ln b) .
$$

Proof. The proof is similar to the proof of Lemma 4.3. So, we omit it.

Lemma 6.3. Let $M={ }_{f} M^{\theta} \times{ }_{b} M^{T}$ be a conformal-twisted product semi-slant submanifold of a g.c.K. manifold $(\bar{M}, J, \omega, g)$. Then, for all $X \in \mathcal{L}\left(M^{T}\right)$, we have

$$
\omega(X)=0 \text {. }
$$

Proof. The proof is similar to the proof of Lemma 4.2. So, we omit it.

Theorem 6.4. Let $M$ be a proper semi-slant submanifold of a g.c.K. manifold $(\bar{M}, J, \omega, g)$. Then $M$ is a locally conformal-twisted product submanifold of type ${ }_{f} M^{\theta} \times_{b} M^{T}$ if and only if for any $X \in \Gamma\left(\mathcal{D}^{T}\right)$ and $V \in \Gamma\left(\mathcal{D}^{\theta}\right)$, we have

$$
\begin{gathered}
\omega(V)=V(\mu), \\
\omega(X)=0, \\
A_{F V} J X-A_{F P V} X=\frac{1}{2}\{\omega(F P V) X-\omega(F V) J X\} \\
-V(\mu) X \sin ^{2} \theta
\end{gathered}
$$

for some function $\mu$.

Proof. Let $M$ be a conformal-twisted product submanifold of the g.c.K. manifold $(\bar{M}, J, \omega, g)$ of type ${ }_{f} M^{\theta} \times_{b} M^{T}$. The equations (6.3) is already obtained in Lemma 6.2 for $\mu=\frac{2}{3} \ln b$. On the other hand, we have $\omega(X)=0$ from Lemma 6.3. Thus, we get (6.4). Now, for any $X \in \mathcal{L}\left(M^{T}\right)$ and $V \in \mathcal{L}\left(M^{\theta}\right)$, we write

$$
A_{F V} J X-A_{F P V} X=\left(A_{F V} J X-A_{F P V} X\right)^{T}+\left(A_{F V} J X-A_{F P V} X\right)^{\theta},
$$

where $\left(A_{F V} J X-A_{F P V} X\right)^{T}$ is the tangent part of $A_{F V} J X-A_{F P V} X$ to $M^{T}$ and $\left(A_{F V} J X-A_{F P V} X\right)^{\theta}$ is the tangent part of $A_{F V} J X-A_{F P V} X$ to $M^{\theta}$. Hence, for 
any $Y \in \mathcal{L}\left(M^{T}\right)$, using (2.2) and (6.1), we have

$$
\begin{aligned}
g\left(A_{F V} J X-A_{F P V} X, Y\right) & =-\omega(V) g(X, Y) \sin ^{2} \theta \\
& +\frac{1}{2}\{\omega(F P V) g(X, Y)-\omega(F V) g(J X, Y)\}
\end{aligned}
$$

from (3.6). Hence, it follows that

$$
\left(A_{F V} J X-A_{F P V} X\right)^{T}=-\omega(V) \sin ^{2} \theta X+\frac{1}{2}\{\omega(F P V) X-\omega(F V) J X\} .
$$

Similarly, for any $U \in \mathcal{L}\left(M^{\theta}\right)$, using (2.3) and (6.2), we have

$$
g\left(A_{F V} J X-A_{F P V} X, U\right)=-g(U, V) X(\ln f)
$$

from (3.7). Since $U \in \mathcal{L}\left(M^{\theta}\right)$ is arbitrary and $f$ depends only on the points of $M^{\theta}$, $X(\ln f)=0$. So

$$
\left(A_{F V} J X-A_{F P V} X\right)^{\theta}=0 .
$$

Since $\mu=\frac{2}{3} \ln b$, by using $(6.6) \sim(6.8)$, we get (6.5).

Conversely, suppose that $M$ is a semi-slant submanifold of a g.c.K. manifold $(\bar{M}, J, \omega, g)$ such that (6.3) (6.5) hold. Then, for any $X \in \Gamma\left(\mathcal{D}^{T}\right)$ and $U, V \in \Gamma\left(\mathcal{D}^{\theta}\right)$, using (6.4) and (6.5), we obtain (3.9). Thus, by Theorem 3.5, the slant distribution $\mathcal{D}^{\theta}$ is totally geodesic. On the other hand, using (6.5), we deduce (3.10). Thus, by Theorem 3.7, the holomorphic distribution $\mathcal{D}^{T}$ is integrable. Let $M^{T}$ and $M^{\theta}$ be the integral manifolds of $\mathcal{D}^{T}$ and $\mathcal{D}^{\theta}$, respectively and let denote by $h^{T}$ and $h^{\theta}$ the second fundamental forms of $M^{T}$ and $M^{\theta}$ in $M$, respectively. Then, for any $X, Y \in \Gamma\left(\mathcal{D}^{T}\right)$ and $V \in \Gamma\left(\mathcal{D}^{\theta}\right)$, using (2.9), we have

$$
g\left(h^{T}(X, Y), V\right)=g\left(\nabla_{X} Y, V\right) .
$$

Here, if we use (6.3) and (6.5), we deduce that

$$
g\left(h^{T}(X, Y), V\right)=-\nabla(\ln b) g(X, Y)
$$

from (3.6). It means that $M^{T}$ is totally umbilic in $M$ with parallel mean curvature vector field $-\nabla(\ln b)$. On the other hand, for any $X \in \Gamma\left(\mathcal{D}^{T}\right)$ and $U, V \in \Gamma\left(\mathcal{D}^{\theta}\right)$, using (2.9), we have

$$
g\left(h^{\theta}(U, V), X\right)=g\left(\nabla_{U} V, X\right) .
$$

Here, using (6.4) and (6.5), we obtain

$$
g\left(h^{\theta}(U, V), X\right)=0
$$

from (3.7). Hence, we conclude that

$$
h^{\theta}(U, V)=0 .
$$

Which means that $M^{\theta}$ is totally geodesic in $M$. Hence, by Remark 4.6 and Remark 4.7, we know that $M$ is conformal-twisted product ${ }_{f} M^{\theta} \times{ }_{b} M^{T}$ of a holomorphic submanifold $M^{T}$ and a slant submanifold $M^{\theta}$ of $M$, where $M^{T}$ is a leaf of $\mathcal{D}^{T}$ and $M^{\theta}$ is leaf of $\mathcal{D}^{\theta}$ and $b$ is a certain twisting function. 


\section{An inequality for conformal-twisted product semi-slant submanifolds in the form ${ }_{f} M^{\theta} \times_{b} M^{T}$}

In this section, we shall establish an inequality for the squared norm of the second fundamental form of a conformal-twisted product semi-slant submanifold in the form ${ }_{f} M^{\theta} \times{ }_{b} M^{T}$, where $M^{T}$ is a holomorphic and $M^{\theta}$ is a slant submanifold of a g.c.K. manifold $(\bar{M}, J, \omega, g)$.

Lemma 7.1. Let $M={ }_{f} M^{\theta} \times_{b} M^{T}$ be a conformal-twisted product semi-slant submanifold of a g.c.K. manifold $(\bar{M}, J, \omega, g)$ and $h$ be the second fundamental form of $M$ in $\bar{M}$. Then we have

$$
\begin{gathered}
g(h(X, Y), F V)=\omega(V) g(X, J Y)+\omega(P V) g(X, Y)-\frac{1}{2} \omega(F V) g(X, Y), \\
g(h(X, U), F V)=0,
\end{gathered}
$$

where $X, Y \in \mathcal{L}\left(M^{T}\right)$ and $U, V \in \mathcal{L}\left(M^{\theta}\right)$.

Proof. The proof is similar to the proof of Lemma 5.1. So, we omit it.

Theorem 7.2. Let $M={ }_{f} M^{\theta} \times_{b} M^{T}$ be a conformal-twisted product semi-slant submanifold of a g.c.K. manifold $(\bar{M}, J, \omega, g)$ and $h$ be the second fundamental form of $M$ in $\bar{M}$. If $h(X, Y) \in \Gamma(\overline{\mathcal{D}})$ for all $X, Y \in \mathcal{L}\left(M^{T}\right)$ and Lee vector field $B$ is tangent to $M$, then $M={ }_{f} M^{\theta} \times_{b} M^{T}$ is a direct product manifold.

Proof. Let $M={ }_{f} M^{\theta} \times{ }_{b} M^{T}$ be a conformal-twisted product semi-slant submanifold of a g.c.K. manifold $(\bar{M}, J, \omega, g)$ and $h$ be the second fundamental form of $M$ in $\bar{M}$. Let $h \in \overline{\mathcal{D}}$ and Lee vector field $B$ is tangent to $M$. Then, for any $X, Y \in \mathcal{L}\left(M^{T}\right)$ and $V \in \mathcal{L}\left(M^{\theta}\right)$, we find

from (7.1), since $\omega(F V)=0$. From (7.3), we find

$$
g(h(X, Y), F V)=\omega(V) g(X, J Y)+\omega(P V) g(X, Y)=0
$$

$$
\omega(V) g(J X, Y)=\omega(P V) g(X, Y) .
$$

In (7.4), if we write $J X$ instead of $X$, we find

$$
-\omega(V) g(X, Y)=\omega(P V) g(J X, Y)
$$

and if we write $P V$ instead of $V$ in (7.5), we find

$$
-\omega(P V) g(X, Y)=\omega\left(P^{2} V\right) g(J X, Y) .
$$

After some calculation, we conclude that from (3.4),

$$
\cos ^{2} \theta \omega(V) g(J X, Y)=\omega(P V) g(X, Y) .
$$

Using (7.4) and (7.7), we have

$$
\sin ^{2} \theta \omega(V) g(J X, Y)=0 .
$$

Since $g(J X, Y) \neq 0$, we find $\omega(V)=0$. Using (6.1), we conclude that twisting function $b$ depends only on the points of $M^{T}$. Then, metric tensor is of the form $g=f^{2} g_{\theta} \oplus b^{2} g_{T}$. So $M$ is direct product of $\left(M^{\theta}, g_{1}\right)$ and $\left(M^{T}, g_{2}\right)$, where $g_{1}=f^{2} g_{\theta}$ and $g_{2}=b^{2} g_{T}$.

Theorem 7.3. Let $M={ }_{f} M^{\theta} \times{ }_{b} M^{T}$ be a conformal-twisted product semi-slant submanifold of a g.c.K. manifold $(\bar{M}, J, \omega, g)$ and the Lee vector field $B$ is tangent to $M$. Then we have

(i) The squared norm of the second fundamental form $h$ of $M$ satisfies

$$
\|h\|^{2} \geq m_{1}\left(1+\cos ^{2} \theta\right) \csc ^{2} \theta\left\|B^{\theta}\right\|^{2}
$$

where $m_{1}=\operatorname{dim}\left(M^{T}\right), m_{2}=\operatorname{dim}\left(M^{\theta}\right)$ and $B^{\theta}$ is tangential part of $B$ to $\mathcal{D}^{\theta}$.

(ii) If the equality sign of (7.9) holds identically, then $M^{\theta}$ is also umbilic in the ambient manifold $\bar{M}$. 
Proof. The squared norm of the second fundamental form $h$ can be written as

$$
\|h\|^{2}=\left\|h\left(\mathcal{D}^{T}, \mathcal{D}^{T}\right)\right\|^{2}+\left\|h\left(\mathcal{D}^{T}, \mathcal{D}^{\theta}\right)\right\|^{2}+\left\|h\left(\mathcal{D}^{\theta}, \mathcal{D}^{\theta}\right)\right\|^{2} .
$$

In view of decomposition (3.3), which can be explicitly written as follows:

$$
\begin{aligned}
\|h\|^{2}= & \sum_{r, s=1}^{m_{1}} \sum_{i=1}^{m_{2}} g\left(h\left(e_{r}, e_{s}\right), e_{i}^{*}\right)^{2}+\sum_{i, j=1}^{m_{2}} \sum_{r=1}^{m_{1}} g\left(h\left(e_{r}, \bar{e}_{i}\right), e_{j}^{*}\right)^{2} \\
& +\sum_{r, s=1}^{m_{1}} \sum_{t=1}^{l} g\left(h\left(e_{r}, e_{s}\right), \hat{e}_{t}\right)^{2}+\sum_{r=1}^{m_{1}} \sum_{i=1}^{m_{2}} \sum_{t=1}^{l} g\left(h\left(e_{r}, \bar{e}_{i}\right), \hat{e}_{t}\right)^{2} \\
& +\left\|h\left(\mathcal{D}^{\theta}, \mathcal{D}^{\theta}\right)\right\|^{2} .
\end{aligned}
$$

Hence, we have

$$
\|h\|^{2} \geq \sum_{r, s=1}^{m_{1}} \sum_{i=1}^{m_{2}} g\left(h\left(e_{r}, e_{s}\right), e_{i}^{*}\right)^{2}+\sum_{i, j=1}^{m_{2}} \sum_{r=1}^{m_{1}} g\left(h\left(\bar{e}_{i}, e_{r}\right), e_{j}^{*}\right)^{2} .
$$

By Remark 5.2 and (7.2) we obtain

$$
\|h\|^{2} \geq \sum_{r, s=1}^{m_{1}} \sum_{i=1}^{m_{2}} g\left(h\left(e_{r}, e_{s}\right), \csc \theta F \bar{e}_{i}\right)^{2} .
$$

Since $\omega(F V)=g(B, F V)=0$, using (7.1), we get

$$
\begin{aligned}
\|h\|^{2} \geq & \csc ^{2} \theta \sum_{r, s=1}^{m_{1}} \sum_{i=1}^{m_{2}} g^{2}\left(e_{r}, J e_{s}\right) \omega^{2}\left(\bar{e}_{i}\right) \\
& +\csc ^{2} \theta \sum_{i, j=1}^{m_{2}} \sum_{r=1}^{m_{1}} \omega^{2}\left(P \bar{e}_{i}\right) g^{2}\left(e_{r}, e_{s}\right) \\
& +2 \csc ^{2} \theta \sum_{i, j=1}^{m_{2}} \sum_{r=1}^{m_{1}} \omega\left(\bar{e}_{i}\right) g\left(e_{r}, J e_{s}\right) \omega\left(P \bar{e}_{i}\right) g\left(e_{r}, e_{s}\right) .
\end{aligned}
$$

Using (2.6), we obtain

$$
\begin{aligned}
\|h\|^{2} \geq & \csc ^{2} \theta \sum_{r, s=1}^{m_{1}} \sum_{i=1}^{m_{2}} g^{2}\left(e_{r}, J e_{s}\right) g^{2}\left(B, \bar{e}_{i}\right) \\
& +\csc ^{2} \theta \sum_{r, s=1}^{m_{1}} \sum_{i=1}^{m_{2}} g^{2}\left(B, P \bar{e}_{i}\right) g^{2}\left(e_{r}, e_{s}\right) \\
& +2 \csc ^{2} \theta \sum_{r, s=1}^{m_{1}} \sum_{i=1}^{m_{2}} g\left(B, \bar{e}_{i}\right) g\left(e_{r}, J e_{s}\right) g\left(B, P \bar{e}_{i}\right) g\left(e_{r}, e_{s}\right)
\end{aligned}
$$

Here, the term

$$
\begin{aligned}
& \sum_{r, s=1}^{m_{1}} \sum_{i=1}^{m_{2}} g\left(B, \bar{e}_{i}\right) g\left(e_{r}, J e_{s}\right) g\left(B, P \bar{e}_{i}\right) g\left(e_{r}, e_{s}\right) \\
& =\sum_{r, s=1}^{m_{1}} \sum_{i=1}^{m_{2}} g\left(B, \bar{e}_{i}\right) g\left(B, P \bar{e}_{i}\right) g\left(e_{r}, J e_{s}\right) g\left(e_{r}, e_{s}\right) \\
& =-\sum_{r, s=1}^{m_{1}} \sum_{i=1}^{m_{2}} g\left(B, \bar{e}_{i}\right) g\left(J B, \bar{e}_{i}\right) g\left(e_{r}, J e_{s}\right) g\left(e_{r}, e_{s}\right) \\
& =-g(J B, B) \sum_{r, s=1}^{m_{1}} g\left(e_{r}, J e_{s}\right) g\left(e_{r}, e_{s}\right)=0,
\end{aligned}
$$


since $g(J B, B)=0$. Thus, we arrive at

$$
\begin{aligned}
\|h\|^{2} \geq & \csc ^{2} \theta \sum_{r, s=1}^{m_{1}} \sum_{i=1}^{m_{2}} g^{2}\left(e_{r}, J e_{s}\right) g^{2}\left(B, \bar{e}_{i}\right) \\
& +\csc ^{2} \theta \sum_{r, s=1}^{m_{1}} \sum_{i=1}^{m_{2}} g^{2}\left(B, P \bar{e}_{i}\right) g^{2}\left(e_{r}, e_{s}\right) .
\end{aligned}
$$

Using Remark 5.2, we find

$$
\begin{aligned}
\|h\|^{2} \geq & \csc ^{2} \theta \sum_{r, s=1}^{m_{1}} \sum_{i=1}^{m_{2}} g^{2}\left(e_{r}, J e_{s}\right) g^{2}\left(B, \bar{e}_{i}\right) \\
& +\csc ^{2} \theta \cos ^{2} \theta \sum_{r, s=1}^{m_{1}} \sum_{i, j=1}^{m_{2}} g^{2}\left(B, \bar{a}_{i}\right) g^{2}\left(e_{r}, e_{s}\right) .
\end{aligned}
$$

Thus, by direct calculation, we obtain the following inequality,

$$
\|h\|^{2} \geq m_{1} \cot ^{2} \theta\left\|B^{\theta}\right\|^{2}+m_{1} \csc ^{2} \theta\left\|B^{\theta}\right\|^{2} .
$$

Rearrange the last inequality, we get the inequality (7.9). If the equality sign of (7.9) holds identically, then we have $h\left(\mathcal{D}^{\theta}, \mathcal{D}^{\theta}\right)=0$ from (7.10). Namely, $h$ vanishes on $\mathcal{D}^{\theta}$. Since $\mathcal{D}^{\theta}$ is a umbilic distribution on $M$, it follows that $M^{\theta}$ is umbilic in $\bar{M}$.

Remark 7.4. Whether the Lee form $\omega$ is exact or not does not change all results in this paper. Thus, these results are also hold for locally conformal Kaehler case. For recent results on the geometry of submanifolds in locally conformal Kaehler ambient space, see $[4,9,12,27,28]$.

Acknowledgment. This work is supported by 1001-Scientific and Technological Research Projects Funding Program of The Scientific and Technological Research Council of Turkey (TUBITAK) project number 119F179.

\section{References}

[1] A. Bejancu, CR-Submanifolds of Kaehler manifold, Proc. Amer. Math. Soc. 69, 135$142,1978$.

[2] A. Bejancu , Semi-invariant submanifolds of locally product Riemannian manifolds, An. Univ. West. Timis. Ser. Mat.-Inform. 22, 1984.

[3] R.L. Bishop and B. O'Neill, Manifolds of negative curvature, Trans. Amer. Math. Soc. 1 (145), 1-49, 1969.

[4] V. Bonanzinga and K. Matsumoto, Twisted product CR-submanifolds in a locally conformal Kaehler space forms, Filomat, 34 (6), 1915-1925, 2020.

[5] B.Y. Chen, Geometry of submanifolds and its applications, Science University of Tokyo, 1981.

[6] B.Y. Chen, Geometry of slant submanifolds, Katholieke Universiteit, Leuven, Leuven 1990.

[7] B.Y. Chen, Geometry of warped product CR-submanifolds in Kaehler manifolds, Monatsh. Math. 133, 177-195, 2001.

[8] B.Y. Chen, Differential geometry of warped product manifolds and submanifolds, World Scientific, 2017.

[9] B.Y. Chen, F. Alghamdi and S. Uddin, Geometry of pointwise semi-slant warped products in locally conformal Kaehler manifolds, arXiv:2011.03997, 2020.

[10] S. Dragomir and L. Ornea, Locally conformal Kähler geometry, Progress in Mathematicsi 155, Birkhäuser, Boston Inc: Boston, MA, 1998.

[11] M. Gutierrez and B. Olea, Semi-Riemannian manifolds with a doubly warped structures, Rev. Mat. Iberoam. 28 (1), 1-24, 2012. 
[12] M.A. Lone, A lower bound of normalized scalar curvature for the submanifolds of locally conformal Kaehler space form using Casorati curvatures, Filomat, 31 (15), 4925-4932, 2017.

[13] K. Matsumoto, Warped product semi-slant submanifolds in locally conformal Kaehler manifolds, Proc. Int. Geom. Cent. 10 (2), 8-23, 2017.

[14] K. Matsumoto, Warped product semi-slant submanifolds in locally conformal Kaehler manifolds II, Proc. Int. Geom. Cent. 11 (3), 27-44, 2018.

[15] M.I. Munteanu, Doubly warped product CR-submanifolds in locally conformal Kähler manifolds, Monatsh. Math. 150, 333-342, 2007.

[16] M.I. Munteanu, A note on doubly warped product contact CR-submanifolds in transSasakian manifolds, Acta Math. Hungar. 116 (1-2), 121-126, 2007.

[17] N. Papaghiuc, Semi-slant submanifolds of a Kählerian manifold, An. Stiint. Univ. Al. I. Cuza Iasi. Mat. (N.S.) 40, 55-61, 1994.

[18] R. Ponge and H. Reckziegel, Twisted products pseudo- Riemannian geometry, Geom. Dedicata, 48, 15-25, 1993.

[19] B. Şahin, Nonexistence of warped product semi-slant submanifolds of Kaehler manifolds, Geom. Dedicata, 117, 195-202, 2005.

[20] B. Şahin, Notes on doubly warped and doubly twisted product CR-submanifolds of Kaehler manifolds, Mat. Vesnik, 59, 205-210, 2007.

[21] B. Şahin, Warped product semi-slant submanifolds of a locally product Riemannian manifold, Studia Sci. Math. Hungar. 46 (2), 169-184, 2009.

[22] H.M. Taştan and S. Gerdan, Doubly twisted product semi-invariant submanifolds of a locally product Riemannian manifold, Mathematical Advances in Pure and Applied Sciences, 1 (1), 23-26, 2018.

[23] H.M. Taştan and M.M. Tripathi, Semi-slant submanifolds of a locally conformal Kaehler manifold, An. Stiint. Univ. Al. I. Cuza Iasi. Mat. (N.S.) Mathematics Tomul LXII, 1 (2), 337-347, 2016.

[24] S. Uddin, On doubly warped and doubly twisted product submanifolds, Int. Electron. J. Geom. 3 (1), 35-39, 2010.

[25] B. Ünal, Doubly warped products, Differential Geom. Appl. 15, 253-263, 2001.

[26] I. Vaisman, On locally and globally conformal Kähler manifolds, Trans. Amer. Math. Soc. 262 (2), 533-542, 1980.

[27] G.E. Vilcu, Ruled CR-submanifolds of locally conformal Kähler manifolds, J. Geom. Phys. 62 (6), 1366-1372, 2012.

[28] G.E. Vilcu, Horizontally conformal submersions from CR-submanifolds of locally conformal Kaehler manifolds, Mediterr. J. Math. 17 (26), 2020.

[29] K. Yano and M. Kon, Structures on Manifolds, World Scientific, Singapore, 1984. 